\title{
Residual life assessment of electricity pylons - a case study
}

\author{
John R Maguire, BSc, PhD, CEng, FICE, FIStructE ${ }^{1 \text {, a }}$ \\ ${ }^{1}$ Lloyd's Register EMEA, Technical Investigations, 71 Fenchurch Street,London, UK \\ ajohn.maguire@Ir.org
}

Keywords: life, structural, assessment, pylons

\begin{abstract}
This case study describes a structural integrity assessment of a $220 \mathrm{kV}$ overhead power line. The line comprises 70 pylons over a distance of approximately $30 \mathrm{~km}$, predominantly in a valley location. The pylons are spaced at intervals of approximately $400 \mathrm{~m}$ and each pylon is approximately $32 \mathrm{~m}$ in height. The line was originally constructed in the 1950's, approximately 50 years prior to the requested structural integrity assessment. This paper describes the independent assessment that was carried out. The review established site-specific safety factors at the time of original design and construction; at the time of the review (2007), accounting for the possible presence of the "Thomasstahl" steel; and in the future, at the anticipated end of pylon life (in 2012).
\end{abstract}

\section{Introduction}

A significant reason behind requesting this particular assessment was the failure of part of a power line in an adjacent country which was considered to exhibit certain similarities with the subject line. Both lines had been partially constructed with steel components which had used the "Thomasstahl process" during steel making, resulting in a steel which has been found to become more brittle than usual with age. Following a high-level risk assessment, a set of visual inspections, materials tests and chemical analyses of the 70 pylons were conducted, which suggested that all 70 were possibly at risk from Thomasstahl-affected steel. It was therefore decided that a more detailed structural integrity assessment (SIA) should be carried out on 12 specific pylons (that had to be structurally modified for other reasons), and that such an assessment be carried out by an independent body - Lloyd's Register EMEA, Technical Investigations. A photograph of a typical pylon is shown in Fig.1. The reasoning behind the structural modifications was excessive sagging of some of the power cables (conductors), due to long term cable creep, which had necessitated the raising up of the lower conductors onto new hangers during 2007, as shown in Fig.1 below.

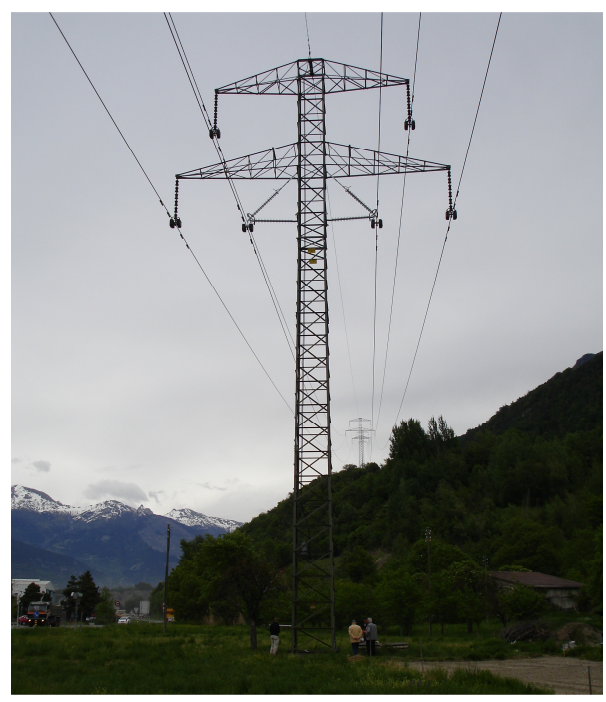

Fig.1. General view of typical pylon 


\section{History}

Following a number of exploratory meetings between the client and Technical Investigations (TID) a scope of work gradually evolved. The client had a requirement not only to assess the structural integrity of the 12 specific pylons but also desired to develop a wider approach to structural integrity assessment and structural condition monitoring that could be used more widely across their grid. During the exploratory meetings it transpired that the existing line had been selected for upgrade, with a new $440 \mathrm{kV}$ line, in approximately 5 years time. Hence the structural assessment would need to bear in mind the relatively short time remaining in the life of the $220 \mathrm{kV}$ pylons.

In view of all the above the TID assessment scope was defined to carry out an independent review of the analyses and tests carried out by the client to date, as well as on the design modification (new lower conductor support arrangements), with a view to establishing the actual safety factor at the time the 12 pylons of interest were originally built; the safety factor in 2007 before the lower conductor support modifications taking into account the suspected presence of Thomasstahl steel; the safety factor in 2007 after the lower conductor support modifications taking into account the suspected presence of Thomasstahl steel; and the expected safety factor in 5 years time (at the anticipated end of life) taking into account the suspected presence of Thomasstahl steel.

As part of the TID activities it was agreed that some supplementary experimental (measurement) and theoretical (analytical) work should be carried out. These supplementary activities comprised member visual inspection, dimensional and thickness measurements; dynamic response analysis of the pylons using a laser-based system; foundation inspections; limited further materials testing; and limited finite element modelling of the pylons.

\section{Approach to assessment}

\section{Methodology}

The pylons may be regarded as "assets" for which the client has a responsibility. As regards "best practice" for asset management there is published guidance available, such as British Standard BS PAS 55 [1]. As regards structural integrity assessment (SIA), which can be thought of as a "snapshot in time", there are codes, standards and best practice available. In the UK the Dynamic Testing Agency (DTA) SIA Primer is a useful reference [2]. As regards structural condition monitoring ISO 16587:2004 [3] is also relevant to the pylons and can be used in a complimentary manner for structural integrity assessment. The principles embodied in all the above-mentioned documents were adopted during the assessment.

\section{Dynamic loading}

The principal loading of the pylons is from the wind, and is therefore dynamic in nature. Snow and ice loading can also be significant but were not considered in detail in this assessment. In reality wind loading is both continuous and random in nature. However, conventional design and assessment codes usually treat wind loads as an equivalent static load, with a dynamic amplification factor either implicitly or explicitly assessed. The available wind data for the site and the wind loading practices adopted by the client in the past dictated that this assessment was carried out on the basis of equivalent steady (quasi-static) loads only.

\section{Safety factors}

Safety factors are used to account for uncertainties in the analysis, design and assessment of structures. Uncertainties arise because parameters such as loads, material properties and structure 
dimensions are treated as constants although in real structures they can vary both within the structure and during its operational life.

Structural design and assessment [2] was originally undertaken by following rules of thumb which were developed on the basis of experience gained over the years and were frequently concerned with the specification of geometric parameters. Subsequent development introduced a stronger theoretical basis and has led to three approaches to the application of "safety factors", namely: - permissible stress (where a safety factor is applied on material resistance);

- factored load (where a safety factor is applied on the applied loading);

- factored stress and load (where partial factors are applied to both materials and loading).

The permissible stress approach, also known as factored stress design, requires the stress level under applied loads to be less than an allowable stress, specified in a code of practice. The allowable stress is defined as the failure stress of the material reduced by a factor of safety whose value is largely determined by experience gained over the years. This has been, historically, an approach favoured in Europe. For mild structural (carbon) steels a material factor of safety is typically of the order of 1.7 to 2.0 [2]. The factored load approach, also known as ultimate load, limit load or load factor method, is one in which the applied loads are increased by a safety factor. Stress levels are required to be less than the failure stress. This has been, historically, an approach favoured in North America. The factored stress and load approach, sometimes called the partial safety factor approach or limit state approach, is a comparatively recent development and aims to achieve a more uniform level of safety by applying safety factors to both the loading on a structure and its resistance. Modern structural Eurocodes [4] have adopted such an approach. Partial factors on loads (typically 1.4 to 1.6) and materials (typically 1.15 on steels) have been chosen to give a factor of safety broadly equivalent to that of permissible stress approaches (e.g. 1.5 x $1.15=1.7$, approximately).

For the purposes of this assessment two of the above three approaches were examined, namely permissible stress and limit state. It is permitted to use lower values of partial safety factors in assessment, as opposed to design, as considerably more information is known or can be obtained than is available at the design stage. The permissible stress assessment approach was based around the corresponding British Standard for steel structures BS 449 [5] and the limit state assessment code based around Eurocodes 1 and 3 [4,6]. Further, in the limit state assessment approach reference will be made to structural reliability, an alternative measure of "safety factor", which will be defined and explained later in this paper.

\section{Limited (critical) locations and failure modes}

In line with the stated scope of work, and the comparative nature of the assessment, this structural integrity assessment was only based on the consideration of areas where critical failures may occur (as opposed to a design check, where all areas of the structure are to be examined). It is noted that a detailed design check had been carried out recently which had given sufficient confidence to limit this assessment to one highly stressed area, namely towards the bottom of the pylons, just above foundation level, where high tensile (and compressive) stresses can occur under wind loading. A low level location was considered to be the most critical and therefore the most relevant to consider in terms of past, present and future overall factors of safety. As implied above, only a predominantly tensile mode of failure was considered - buckling (compressive) failure appeared to be a less critical case for these particular pylons, on the basis of the bracing layout and general robustness of the structure. However buckling failures have been known to occur on other pylon types. 


\section{Data collected prior to safety factor determination}

The following paragraphs summarise the data collected and analyses carried out prior to the evaluation of safety factors and structural reliability.

\section{Member visual inspection, dimensional and thickness measurements}

During site visits the 12 pylons were inspected visually, in terms of: assessing the general condition of above-ground elements and visible parts of the foundations; checking that all members and bolts were present in comparison to the design drawings; assessing visually that all the bolts were secure; and assessing that the paintwork was in good condition and that there was no excessive corrosion. According to the design drawings, the four main legs of each pylon, just above foundation level, should have been angle sections of leg length $120 \mathrm{~mm}$, leg thickness $10 \mathrm{~mm}$, at the four corners of a square of dimension $2 \mathrm{~m} \times 2 \mathrm{~m}$. It was found that both the leg lengths and plan dimensions accorded closely with the design dimensions, but that the leg thicknesses were often in excess of the minimum specified - typically $11 \mathrm{~mm}$, rather than $10 \mathrm{~mm}$. This thickness variation was taken into account.

In general the condition of the steel elements, concrete foundations and bolts appeared good. There were no cracks apparent in the above-ground steel elements, little cracking in the concrete foundations (although a few areas needed some patch repair) and all bolts were present and appeared to be secure. In the vicinity of a few of the weldments just above the concrete foundations there was some evidence of weld-related cracking, which the client planned to monitor carefully in future.

\section{Dynamic response analysis}

During site visits a specialist laser measurement company was employed by the client, under TID supervision, to carry out a number of dynamic response measurements of the pylons under various wind conditions. The main objective of the testing was to determine experimental frequencies of vibration of the pylons, for later use in the tuning of finite element models of the pylons. Although the readings were difficult to interpret, as a result of being single point measurements, there did seem to be modes of vibration clearly present, particularly around 1.2 to $2.2 \mathrm{~Hz}$ and around 4 to $6 \mathrm{~Hz}$. A correlation exercise was carried out, relating conductor span length to measured frequency which indicated that (generally) as the mass carried (conductor length) increased then the natural frequency decreased, as would normally be expected.

\section{Foundation inspections}

During the site visits it was observed that a few of the foundations were of different (larger) size than indicated on the drawings, and also it was only possible to observe the top surfaces of the foundations. With this in mind a limited number of excavations were carried out to further ascertain foundation condition. From the photographs, and from close visual inspections, it could be seen that the foundations were generally in good condition and as a result the foundations could be confirmed as less critical locations than the lower parts of the four pylon legs with regard to safety factor assessment.

\section{Limited further materials testing}

The client was able to supply in advance a limited amount of materials data from their own testing campaign. Opportunity was taken to collect further samples from one pylon to carry out some confirmatory independent testing. The material tests undertaken at ambient temperature indicated low carbon mild steel with tensile strength in the region of $360 \mathrm{~N} / \mathrm{mm}^{2}$ and good ductility, for the particular samples tested. As regards Young's modulus, room temperature values of between 185 and $196 \mathrm{kN} / \mathrm{mm}^{2}$ were measured. 


\section{Finite element modelling}

TID prepared a number of preliminary finite element models of the pylon structures. The purpose of these analyses included: to give improved understanding of the static and dynamic characteristics of the pylons; to establish "in service " characteristics as opposed to design assumptions by comparison with the results from the dynamic response measurements; and to confirm and give further confidence in the modelling carried out by others. It was expected, as a result of the limited modelling process which did not fully model the conductor cable catenary stiffness, that some differences might exist between measured and predicted frequencies, particularly for the along line and torsion modes.

\section{Comparison of results from dynamic response analysis and finite element modelling}

A numerical comparison of the measurement results and the theoretical predictions may be expressed as follows.

\begin{tabular}{|l|c|c|}
\hline Mode description & $\begin{array}{c}\text { Experimental (dynamic response) } \\
\text { measured data }\end{array}$ & $\begin{array}{c}\text { Theoretical (finite element } \\
\text { analysis) predictions }\end{array}$ \\
\hline Mode 1 - bending & $1.3 \mathrm{~Hz}$, mode shape not measured & $1.2 \mathrm{~Hz}$, bending at right angle \\
\hline Mode 2 - bending & $1.7 \mathrm{~Hz}$, mode shape not measured & $1.4 \mathrm{~Hz}$, bending along line \\
\hline Mode 3 - torsion & $1.9 \mathrm{~Hz}$, mode shape not measured & $2.5 \mathrm{~Hz}$, torsion \\
\hline
\end{tabular}

Table 1. Comparison of the measurement results and the theoretical predictions

An apparently reasonable correlation may be seen, bearing in mind that the experimental mode shapes were not measured (limitation of the laser technique used), and that the finite element modelling was of a preliminary nature. Further work was suggested to the client to improve the reconciliation between the experimental and theoretical data.

\section{Assumptions and definitions relating to structural integrity}

In carrying out a structural integrity assessment (SIA) it is necessary to make certain assumptions, which are explicitly set out below. The terminology used here, and elsewhere in this paper, is selected to be as consistent as practical with Eurocode 1, the head document of the structural Eurocode suite [4].

\section{Assumptions}

It was assumed that:

- the original choice of pylon structural arrangement and design was made by appropriately qualified and experienced personnel at the time of the original design (which appears to have been the case, from the limited records available);

- the execution (design and build) was carried out by personnel of appropriate skill and experience (which appears to have been the case as the pylons appear to have "stood the test of time" to date);

- adequate supervision and quality control was provided during the original execution of the work (which appears to have been the case);

- the pylons have been, and will continue to be, adequately maintained (the client has an ongoing maintenance responsibility).

\section{Requirements and Reliability}

The pylons should [4] be designed, executed and maintained in such a way that, during their intended and remaining life, with an appropriate degree of reliability and in an economic way, they will: remain fit for use under all expected actions; have adequate structural resistance, serviceability and durability; not be damaged by events such as impacts or consequences of human errors to an extent disproportionate to the original cause (robustness requirement). 


\section{Basic variables used in structural assessment}

This section considers in more detail the basic variables used in this SIA, namely the loads (actions) applied to the pylons; material properties; and geometric data. The materials and geometry are used together to determine the capacity (structural resistance) of the pylons. In particular a stress (strength) calculation was carried out at the bottom of a typical pylon, of the form $\mathrm{S}=-(\mathrm{F} / \mathrm{A})+/-(\mathrm{M} / \mathrm{Z})$, where $\mathrm{S}$ is the calculated stress; $\mathrm{F}$ is the axial (vertical) load; $\mathrm{A}$ is the area of the four pylon legs taken together; $\mathrm{M}$ is the wind moment applied to the pylon foundation; $\mathrm{Z}$ is the section modulus of the four pylon legs taken together. The negative sign "_. indicates stresses in a compressive sense, "+" indicates tension. By comparing the calculated stress, S, to the allowable, Sa, the available factor of safety may be determined. The left hand side of the equation (S) represents the "capacity" (or resistance) of the structure. The right hand side (-F/A +/- M/Z) represents the "load" (or demand) on the structure.

\section{Loads}

The principal loads to be considered in this SIA were:

- permanent actions, $\mathrm{G}$ - primarily the self weight of each pylon and the weight of conductors (power cables) carried, which is represented by (F/A) in the above equation;

- variable actions, Q - primarily wind loads. Excluded from this SIA are (variable) snow loads although significant in nature they are considered to be of such a magnitude and sense (causing primarily compressive stresses at the tower base) as to be of secondary interest to this SIA. Ice loads were another possible consideration, but were excluded from this SIA for reasons given earlier. The wind load effects are represented by $(\mathrm{M} / \mathrm{Z})$ in the above equation;

- accidental actions, A - such as fire and earthquake. Such loads were excluded from this SIA, as were accidental torsions, arising from conductor cable breaks. Accidental torsion, although non-trivial in nature, was considered to be of secondary interest to this SIA.

Permanent actions, G, are considered as "static" in nature, as they do not cause any significant accelerations of the pylons or their members. Variable actions, Q, are considered as "dynamic" in nature. As stated earlier dynamic effects of actions are often considered as quasi-static, by increasing the magnitude of the static actions, which was the approach generally adopted in this SIA.

\section{Characteristic values of loads}

The most important representative value of a load, $\mathrm{F}$ (axial, or $\mathrm{M}$, moment), is the characteristic value $\mathrm{F}_{\mathrm{k}}$ (or $\mathrm{M}_{\mathrm{k}}$ ). Depending on the available data and experience, this value may be specified as a mean, upper or lower value or a nominal value (which does not refer to any statistical distribution). There is often considerable lack of relevant statistical data concerning various loads and consequently the determination of a representative value involves not only the evaluation and analysis of available observations and experimental data but also some subjective assessment (judgement).

As regards permanent action, $G$, the self-weight of each pylon and conductor contribution, there were two sources of data as follows:

(i) original pylon drawings containing weight data;

(ii) finite element models which can be used together with assumed steel densities to check/confirm the values from (i).

Variability in permanent action is usually assumed to be relatively small [4]. Hence in the case of self-weight a single value per pylon was adopted, and this value $G_{k}$ (equal to $F_{k}$ in this case) can be assumed to be the mean value, calculated on the basis of nominal dimensions and mean unit mass. 
As regards variable actions, $Q$, the wind load applied to each pylon, there were a variety of data sources. At the time of original design and construction the usual practice was to apply a quasi-static pressure evenly over the height of between 50 and $100 \mathrm{~kg} / \mathrm{m}^{2}\left(500\right.$ to $\left.1000 \mathrm{~N} / \mathrm{m}^{2}\right)$ depending on the particular surface profile. It was noted that there was an equivalence between wind speeds and pressures as follows: wind pressure of $500 \mathrm{~N} / \mathrm{m}^{2}$ results from wind speed of $103 \mathrm{~km} / \mathrm{h}(28.6 \mathrm{~m} / \mathrm{s})$; wind pressure of $750 \mathrm{~N} / \mathrm{m}^{2}$ results from wind speed of $122 \mathrm{~km} / \mathrm{h}(33.9 \mathrm{~m} / \mathrm{s})$; and wind pressure of $1000 \mathrm{~N} / \mathrm{m}^{2}$ results from wind speed of $146 \mathrm{~km} / \mathrm{h}(40.6 \mathrm{~m} / \mathrm{s})$.

As an overall load the quasi-static pressure was converted to a wind overturning moment, $\mathrm{M}$, of $1408 \mathrm{kNm}$ at the level just above the foundation (the critical location in terms of this SIA). This "original" wind load, before modification, was considered to be an upper bound value, and was checked/confirmed via an alternative calculation. However since the time of the original design: understanding of wind loading has advanced, in particular the variation of wind speed with height and knowledge of drag coefficients relating to lattice towers; wind loading practices in the country under consideration have changed slightly and now specify pressures of 500 to $1200 \mathrm{~N} / \mathrm{m}^{2}$ for pylons up to $30 \mathrm{~m}$ in height and higher values for taller pylons; statistical data has become available from a nearby meteorological station covering the last 10 years; and probabilistic approaches to wind loading have developed $[4,6,7]$.

With these changes and developments it was therefore possible to estimate an updated overturning moment, based primarily on statistical analysis of the 1996-2006 data set. A location-specific wind speed for this assessment was decided to be $19.7 \mathrm{~m} / \mathrm{s}$ for a 10 minute mean (at a height of $10 \mathrm{~m}$ ) and $32.7 \mathrm{~m} / \mathrm{s}$ for a 1 second gust. Neither a 10 minute mean or a 1 second gust were considered appropriate for the assessment of a pylon structure - a 5 second mean would appear to be more appropriate [7]. To convert from a 1 second gust to a 5 second mean a factor of 0.9 was chosen. Based on such an analysis the contemporary wind overturning moment was estimated as $1690 \mathrm{kNm}$, both before and after support arm modification.

\section{Materials}

In design calculations the properties of materials, in this case mild carbon steel, are represented by characteristic values, which correspond to a prescribed probability of not being infringed. Usually the lower value of the material property is unfavourable and the $5 \%$ (lower) fractile is then considered. In assessment, in contrast, the actual/most likely/mean value is often used. Also it is noted that stiffness parameters, such as those used in finite element models, are normally defined as mean values.

\section{Characteristic values of materials}

The limited test data available suggested an elastic limit stress, for the leg material, in the range $321-381 \mathrm{~N} / \mathrm{mm}^{2}$, with a corresponding mean of $358 \mathrm{~N} / \mathrm{mm}^{2}$ and a $5 \%$ (lower) fractile value of 315 $\mathrm{N} / \mathrm{mm}^{2}$. Data from other similar pylons suggested mean values ranging from approximately 270 to $330 \mathrm{~N} / \mathrm{mm}^{2}$ in most cases, depending on material type.

For the purpose of this assessment it was assumed, based on the limited test data, that: original design yield stress $=315 \mathrm{~N} / \mathrm{mm}^{2}$ (unfactored); and contemporary assessment yield stress $=358$ $\mathrm{N} / \mathrm{mm}^{2}$ (unfactored).

It is significant to note that the original calculations indicated an allowable, factored, stress (presumably compressive) of $173 \mathrm{~N} / \mathrm{mm}^{2}$ - this allowable may be compared to:

(a) value of $315 /(1.7$ to 2.0$)=157-185 \mathrm{~N} / \mathrm{mm}^{2}$, average $171 \mathrm{~N} / \mathrm{mm}^{2}$, where (1.7 to 2.0$)$ is the type of safety factor described above;

(b) "lower bound" value of $179 \mathrm{~N} / \mathrm{mm}^{2}$;

(c) typical values from British Standards [5] which suggests $165 \mathrm{~N} / \mathrm{mm}^{2}$ for combined tension and bending allowable stresses. 
In view of the above, and in view of the relatively small test data set available, it was considered appropriate to assign the characteristic material properties as follows: original [lower bound] design yield stress in tension $=165 \times 1.7=280 \mathrm{~N} / \mathrm{mm}^{2}$; contemporary (mean) assessment yield stress $=358$ $x(280 / 315)=318 \mathrm{~N} / \mathrm{mm}^{2}$. The $318 \mathrm{~N} / \mathrm{mm}^{2}$ value is considered to include for Thomasstahl effects. Although Thomasstahl effects appear to have a significant influence on ductility, and the shape of the stress/strain curve the effect on (elastic) yield stress appears to be minimal. In view of the generally robust nature of the pylons - discussed above - the pylons may be considered as "damage tolerant" and hence the significance of Thomasstahl brittleness may not be as great as thought by some others.

\section{Geometry}

In design calculations the geometric data should be represented by their characteristic values, which usually correspond to the design dimensions, which are the nominal values. Hence for design, the pylon leg dimensions just above the foundation were used. However in assessment the actual, mean, values may be used. Also some allowance was made for bolt holes, which reduced section thickness locally.

\section{Stress (strength) calculation - permissible stress and limit state approaches}

Calculations were carried out for the original design factor of safety, "target" factor of safety, contemporary assessment factor of safety before modification, contemporary assessment factor of safety after modification, and assessment at end of life circa 2012 assuming that regular inspection and maintenance occurs. It was not expected that loads, materials or geometry would alter by any more than $+/-2 \%$ in the 5 year period 2007 to 2012 .

\section{Discussion}

\section{Safety factors and wind load contribution}

Table 2 gives a tabular summary of the calculated safety factors. It may be seen that: actual safety factors are in excess of the minimum required in all cases; and that contemporary (2007) and future (2012) assessments suggest that adequate factors of safety exist for the modified pylons. For the desired level of structural reliability - "normal", as explained above - the factors of safety appear adequate; and the apparent reduction in safety factors from the original design to the present day is primarily due to the relatively higher assessment wind loadings that have been assumed in 2007 .

\begin{tabular}{|l|c|c|c|c|}
\hline Factor of safety & $\begin{array}{c}\text { Permissible } \\
\text { stress minimum }\end{array}$ & $\begin{array}{c}\text { Permissible stress } \\
\text { actual }\end{array}$ & $\begin{array}{c}\text { Limit state } \\
\text { minimum }\end{array}$ & $\begin{array}{c}\text { Limit state } \\
\text { actual }\end{array}$ \\
\hline At initial design stage & 1.7 & $\begin{array}{c}1.93 \\
(+13 \%)\end{array}$ & 1.0 & $\begin{array}{c}1.09 \\
(+9 \%)\end{array}$ \\
\hline $\begin{array}{l}\text { 2007 assessment, before } \\
\text { modification, including } \\
\text { Thomasstahl }\end{array}$ & 1.7 & $\begin{array}{c}1.80 \\
(+6 \%)\end{array}$ & 1.0 & $\begin{array}{c}1.02 \\
(+2 \%)\end{array}$ \\
\hline $\begin{array}{l}\text { 2007 assessment, after } \\
\text { modification, including } \\
\text { Thomasstahl }\end{array}$ & 1.7 & $\begin{array}{c}1.80 \\
(+6 \%)\end{array}$ & 1.0 & $\begin{array}{c}1.02 \\
(+2 \%)\end{array}$ \\
\hline $\begin{array}{l}\text { End of life (2012) } \\
\text { assessment, after } \\
\text { modification, including } \\
\text { Thomasstahl }\end{array}$ & 1.7 & $\begin{array}{c}1.76 \\
(+4 \%)\end{array}$ & 1.0 & $\begin{array}{c}1.00 \\
(+0 \%)\end{array}$ \\
\hline
\end{tabular}

Table 2. Calculated safety factors

The reader is reminded that the above assessment factors of safety are based on site-specific wind records, which have been shown to locally reduce wind speeds (and hence wind loads) in comparison to other parts of the country where the pylons were located, but this has been offset by the 
requirements of modern wind load design codes which appear to call for higher wind loads $(+20 \%$ in the country, based on increase from 1000 to $1200 \mathrm{~N} / \mathrm{m}^{2}$ over the last 50 years). Other sites, where pylons may be located in more wind-onerous locations, may not be as fortunate when considered for assessment in this manner. The reader is also reminded that this assessment is limited to one critical area (towards the lower part of the pylon structure), under what has been considered to be the most onerous loading case (extreme wind, with no coincident snow or ice effects) - a more detailed design check had been carried out by a separate consultant, using a basic wind pressure of $500 \mathrm{~N} / \mathrm{m}^{2}$, which appeared also to show that the pylons have an adequate margin of safety, when all the structural elements are considered.

It is also notable that the largest contributors to stress towards the bottom of the pylon were:

- wind forces rather than lattice tower self weight loads - the stress contribution ratio is in excess of 10 to 1 ;

- wind forces applied via the conductors rather than wind loads via the lattice tower structure - the conductor wind bending moment is approximately twice that due to the tower structure wind bending moment.

These ratios suggest that, in future assessments of other power lines of this type, due consideration be given to accurate assessment of conductor diameter and length, localised drag coefficients, wind speed and resultant wind load applied to the pylons. It is noted that, in winter conditions, conductors may increase in diameter due to the effects of snow and/or ice, and this will have a consequence for possible (increased) wind loading. However, it may not be reasonable to consider that both maximum wind load and maximum conductor diameter (increased by snow and/or ice) occur simultaneously.

\section{Inspection and maintenance}

As regards inspection and maintenance, an appropriate level should be selected, according to [4], depending on:

- required reliability, in this case "normal";

- risk to life, and economic and social losses, in this case "medium to low";

- consequence of failure, in this case "medium to low".

In the terminology adopted by the Eurocode 1 [4] this corresponds to consequence class CC2, reliability class RC2, which together dictate a need for inspection class IL2, normal inspection, which means "inspection in accordance with the procedures of the organisation". As such, no independent third party inspection is deemed necessary in this case.

\section{Experimental and theoretical data}

Although a certain degree of apparent correlation exists between the experimental (dynamic response) and theoretical (finite element) data as described above, it was considered that this correlation could and should be improved by further work. In particular:

(a) a more sophisticated set of modal tests could yield experimental mode shapes in addition to mode frequencies. There would also be value in taking coincident strain measurements, particularly at a pylon close to the local weather station;

(b) a more sophisticated finite element model, with an appropriate catenary stiffness representing better the conductor (cable) contributions, could yield more accurate modal frequencies and shapes. 


\section{Conservatisms and uncertainties in this SIA}

Permissible-stress-based safety factors have been shown by this SIA to be at least 1.76 to 1.93 (Table $2)$. In reality, for certain of the 12 pylons, the safety factors will be slightly higher, since the actual conductor lengths carried ( 318 to $383 \mathrm{~m}$ ) are less than the assessment value $(400 \mathrm{~m})$. For a "383 m pylon" the safety factor will be fractionally higher than 1.76 to 1.93 . For a "318 m pylon" the safety factor will be significantly (of the order of 10-20\%) higher. However it is considered inappropriate to concentrate too much on relative variations in conductor length, when the magnitude of the wind loading has been shown to contain a relatively much higher degree of variation and uncertainty. It is worth repeating that in future assessments of other power lines due consideration be given to accurate assessment of conductor diameter and length, localised drag coefficients, wind speed and resultant wind load applied to the pylons.

\section{Conclusions}

An independent review has been carried out of analyses and tests conducted by the client and others, relating to 12 pylons which had been modified with new lower conductor support arrangements. This review has established site-specific safety factors at the time of original design and construction; at the time of assessment (2007), before and after structural modification, and accounting for the possible presence of Thomasstahl steel; and in the future (2012), at the anticipated end of pylon life, again accounting for the possible presence of Thomasstahl steel.

The safety margins, in excess of 1.7 on a permissible stress basis, appear adequate for the 12 pylons assessed at the specific site, until the end of life, provided that regular inspection and maintenance activities are carried out within the framework of an appropriate company quality management system.

\section{References}

[1] British Standards Institution. 'PAS 55 - Asset Management'. www.bsi-global.com.

[2] Dynamic Testing Agency. 'Primer on Best Practice in Dynamic Testing'. ISBN 1899630163.

[3] International Standards Organisation. 'ISO16587:2004 - Condition monitoring of structures'. www.iso.org.

[4] Eurocode 1, Basis of Structural Design. 'Designer's Guide to EN 1990'. H Gulvanessian, J-A Calgaro and M Holicky. Thomas Telford Ltd, 2002. ISBN 07277730118.

[5] British Standards Institution. 'BS 449 - Steel structures'. www.bsi-global.com.

[6] Eurocode 1, Actions on Structures. 'Designer's Guide to EN 1991-4'. Wind Actions by N Cook. Thomas Telford Ltd, 2007. ISBN 978-0-7277-3152-4.

[7] British Standards Institution. 'CP3: Chap V: Part 2 - Wind loads'. www.bsi-global.com. 